\title{
Laboratory training in silicon photonics for undergraduate and graduate students
}

\section{Azad Siahmakoun}

Azad Siahmakoun, "Laboratory training in silicon photonics for undergraduate and graduate students," Proc. SPIE 11143, Fifteenth Conference on Education and Training in Optics and Photonics: ETOP 2019, 1114313 (2 July 2019); doi: 10.1117/12.2523580 


\title{
Laboratory Training in Silicon Photonics for Undergraduate and Graduate Students
}

\author{
Azad Siahmakoun \\ Department of Physics and Optical Engineering \\ Rose-Hulman Institute of Technology \\ Terre Haute, Indiana USA 47803 \\ siahmako@rose-hulman.edu
}

\begin{abstract}
We will discuss laboratory experiments in silicon-photonics developed for senior and graduate level students. The learning objectives for this laboratory includes basic microfabrication techniques in cleanroom, understanding design and process flow, fabrication and testing of silicon waveguides and Mach-Zehnder interferometers on a silicon-on-insulator (SOI) wafer. We will also discuss a typical team project outcomes and examples of experimental work and report by student group. Additionally, we will comment on student's learning and skill set acquired through this introductory hands-on project-based silicon photonics class.
\end{abstract}

Keywords: Silicon Photonics, Optical Engineering, SOI Waveguide, MZI Modulator

\section{INTRODUCTION}

We teach an introductory course in silicon-photonics that covers basic properties of silicon waveguides; the essentials of silicon-on-insulator (SOI) waveguide fabrication (WGs); dispersion and propagation loss in Si WGs; analysis and design of various passive silicon devices such as strip and rib waveguides [1], directional couplers, Y-branch splitter/combiner, Mach-Zehnder interferometer (MZI); design and modeling of Bragg gratings and grating couplers for light input/output to/from devices and circuits; develop matrix method analysis in order to determine the transfer function of passive devices; modeling and characterization a Mach-Zehnder silicon modulator [2]; use free shareware KLayout to make device and integrated photonic circuit (PIC) layouts for E-beam lithography laboratory portion of the course.

This introductory course with laboratory in silicon-photonics is offered at senior and graduate level. Learning objectives for this laboratory includes basic skills for microfabrication techniques in the cleanroom such as oxidation, thin film metal deposition, photolithography, wet and dry etch; understanding design and process flow, fabricating and testing of silicon waveguides and Mach-Zehnder interferometers (MZI) on a silicon-on-insulator (SOI) wafer. This is a research based learning class for senior and graduate students on silicon-photonics ( $\mathrm{SiPh}$ ) design, fabrication, and testing where they are given both theoretical and laboratory projects to do.

\section{FABRICATION}

The first part of lab is to fabricate an SOI wafer using dry oxidation followed by PECVD or sputtering processes in the cleanroom. Table 1shows the process flow for fabrication of an SOI wafer (see Figure 1).

Fifteenth Conference on Education and Training in Optics and Photonics: ETOP 2019, edited by Anne-Sophie Poulin-Girard, Joseph A. Shaw, Proc. of SPIE Vol. 11143, 1114313 - (C) 2019

SPIE, ICO, IEEE, OSA · CCC code: 0277-786X/19/\$18 · doi: 10.1117/12.2523580 
Table 1: Process flow for fabricating an SOI based thermos-optic MZI.

\begin{tabular}{|c|c|c|}
\hline $\mathrm{Si}$ & $\mathrm{Si}_{3} \mathrm{~N}_{4}$ & $\mathrm{SiO}_{2}$ \\
\hline $\begin{array}{l}\text { 1. Choosing a bare silicon p-type }\{111\} \\
\text { wafer with } 4 \text { inch in diameter and RCA } \\
\text { clean }\end{array}$ & & \\
\hline $\begin{array}{l}\text { 2. Wet oxidation for } 180 \text { minutes using } \\
\text { wet oxidation Furnace }\end{array}$ & & \\
\hline $\begin{array}{l}\text { 3. Depositing about } 1 \mu \mathrm{m} \text { of silicon } \\
\text { nitride on top of the silicon dioxide using } \\
\text { PECVD or sputter } 0.3 \mu \mathrm{m} \text { of } a-\mathrm{Si}\end{array}$ & & \\
\hline
\end{tabular}

The implemented process flow for a Si device fabrication includes PMMA resist spin coating, e-beam lithography, development, and $\mathrm{Si}$ etch with $\mathrm{XeF}_{2}$ as follows in Table 2.

Table 2. Process flow for E-beam lithography

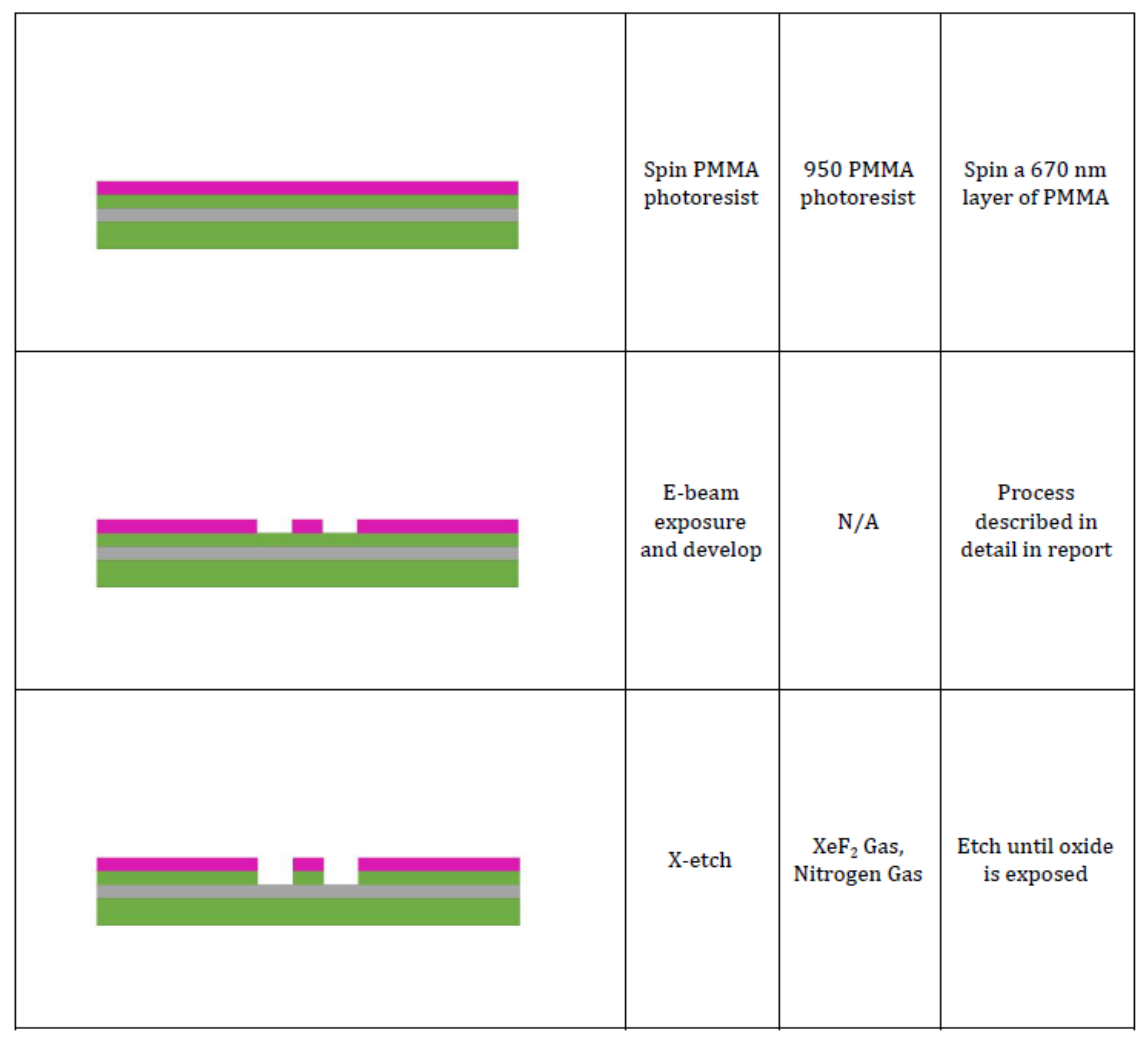



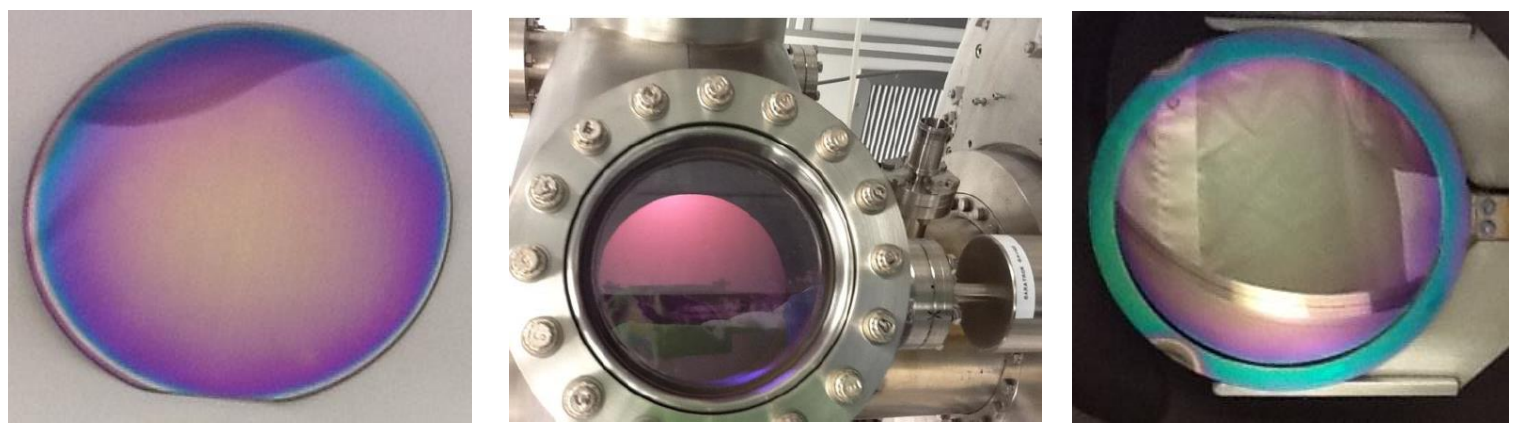

Figure 1. Silicon wafer after the oxidation (left) and the sputtering processes to produce amorphous silicon on oxide (right). Middle image is the plasma glow during sputtering.
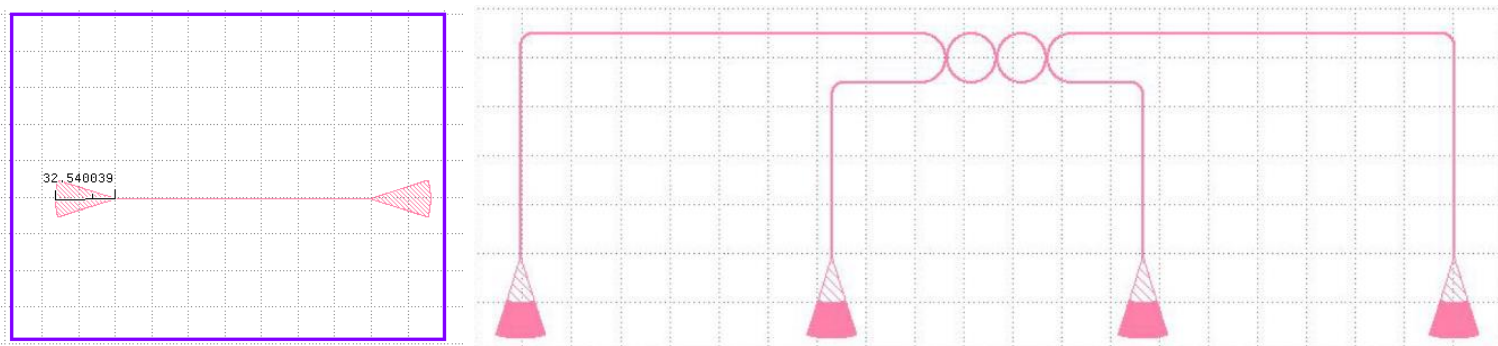

Figure 2. The KLayout design for straight and bend waveguides including ring resonators and grating couplers.

To produce silicon waveguides using the e-beam lithography with SEM the process defined in the NPGS software was followed. The waveguide pattern is a direct-write e-beam onto the photoresist. To determine the proper exposure time a varying dosage of $250,500,750$, and $1000 \mu \mathrm{C} / \mathrm{cm}^{2}$ were irradiated. When the photoresist received a low dosage of $250 \mu \mathrm{C} / \mathrm{cm}^{2}$ the resulting patterns were faint after development as can be seen in Fig.3.

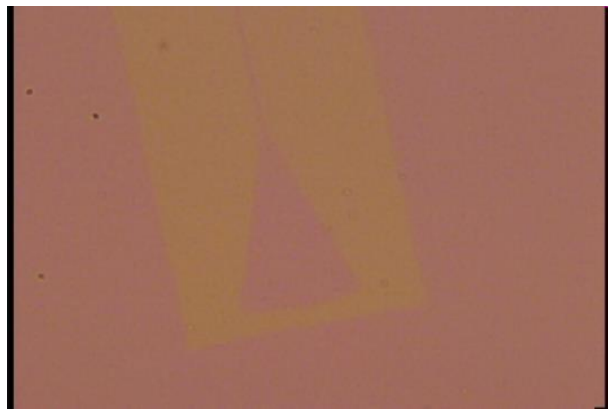

Figure 3. The low dose exposure by e-beam produces patterns.

However, when exposures at varying high measures of e-beam current were performed at values of 500, 750 , and $1000 \mu \mathrm{C} / \mathrm{cm}^{2}$ respectively. The resulting patterns written at these exposures from left to right 1000 $\mu \mathrm{C} / \mathrm{cm}^{2}, 750 \mu \mathrm{C} / \mathrm{cm}^{2}$, and $500 \mu \mathrm{C} / \mathrm{cm}^{2}$ are shown in Fig.4. Based on and their observation by microscope 20x the etched regions appear (a) to have exposed oxide layer, (b) to be partially through the device layer of the SOI, and (c) to have barely been etched past the resist layer. 


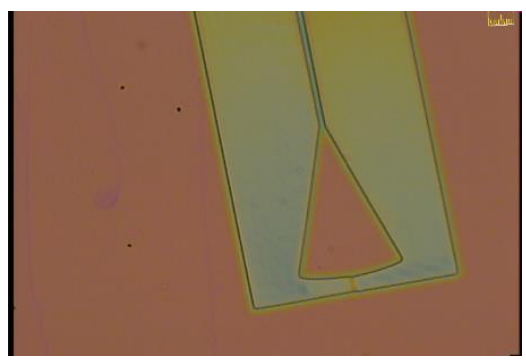

(a)

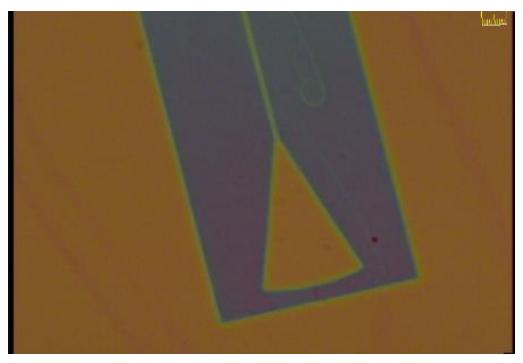

(b)

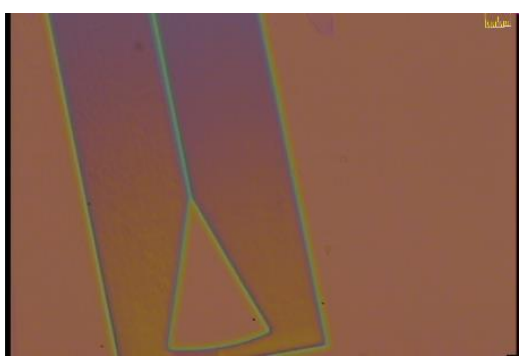

(c)

Figure 4. Waveguides produced at high dosages after silicon $X$-etch. The estimated dosage for (a) is 1000 $\mu \mathrm{C} / \mathrm{cm}^{2}$, (b) is $750 \mu \mathrm{C} / \mathrm{cm}^{2}$, and (c) is $500 \mu \mathrm{C} / \mathrm{cm}^{2}$.

\section{CHARACTERIZATION OF SILICON DEVICES}

Some of the SiPh devices that had been designed and fabricated by students in order to determine quality of manufactured passive devices. A proper testing procedures was used for coupling light into photonic devices. An example of the intricate setup is shown in Figure 5. A set of 2 cameras are used to align the fiber array to the Si die input and output ports. The camera, pointed at an angle, was used to find and align the fiber array to the devices. The horizontal camera was used to bring the fiber array as close to the chip as possible to reduce beam divergence in the air between the array and the chip (see Fig.6).

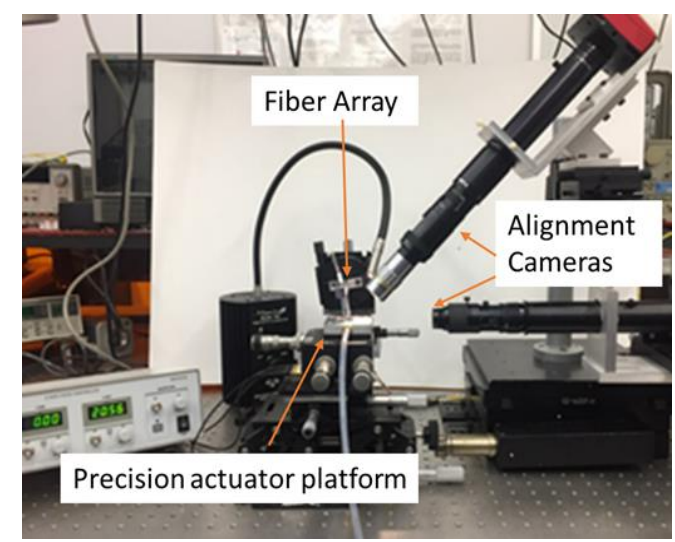

Figure 5. The testing setup used to couple light into a SiPh device. A fiber array is oriented at $8^{\circ}$ with respect to the normal to the surface of the Si die and the fibers are spaced in the array such that they align with the inputs and outputs of the Si devices.

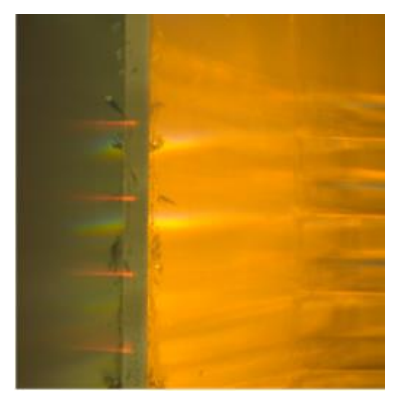

Figure 6. An image of a roughly aligned fiber array to a set of grating coupler input and output channels. 
The setup used to test the photonic devices includes an SOA for amplifying a DFB laser signal, before inserting it into a photonic device. Then measuring the output using an optical spectrum analyzer.

To couple light into photonic devices, the standard spacing between optical inputs and outputs is supposed to match fiber array input and output optical fibers. One can see that the grating couplers are roughly spaced by the same distance as the fibers in the array in Figure 6. However, students managed to couple light into one of the fabricated Si devices. Yet, light coupling came with significant loss as shown in Figure 7(a).

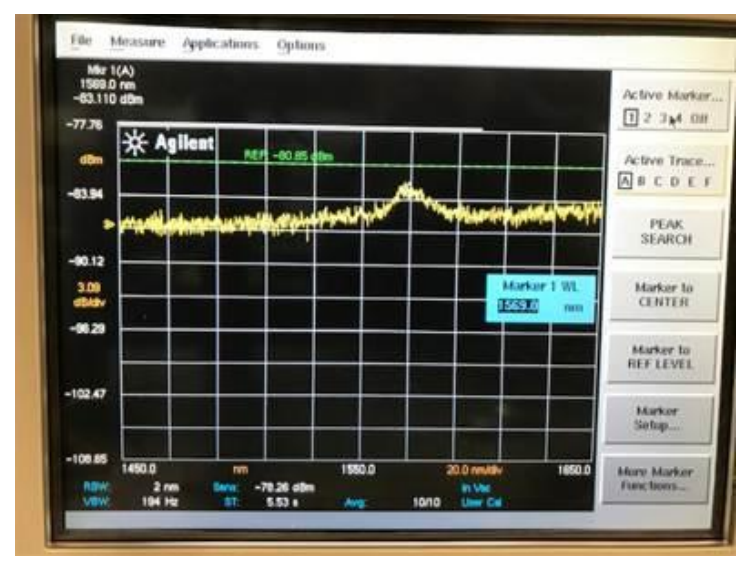

(a)

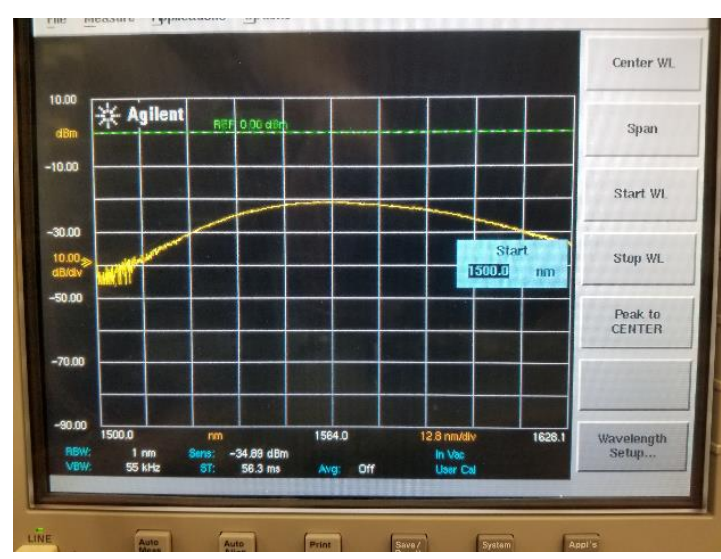

(b)

Figure 7. Optical spectrum analyzer output after measuring (a) the output of a ring resonator and (b) the output of the broadband laser source. The laser source is centered at approximately $1564 \mathrm{~nm}$ with a $20 \mathrm{dBm}$ insertion loss. The resonance peak of the ring resonator is at about $1570 \mathrm{~nm}$ where the output signal was measured at approximately $-80 \mathrm{dBm}$.

Unfortunately, it is very difficult to couple light into a device on the Si die via the fabricated grating couplers and the fiber array and the output signal power was exorbitantly low. This is likely due to the testing set up being difficult to align and that the actual efficiency of the coupler devices on the chip is unknown.

\section{ACKNOWLEDGMENTS}

Author would like to thank cleanroom technician Brian Fair and all my students who help with the fabrication and testing processes of this project, in particular: James Dilts, Simon Tsaoussis, Gavin La Rue, and Joseph Porter.

\section{REFERENCES}

[1] Lukas Chrostowski and Michael Hochberg, "Silicon Photonics Design from Devices to Systems," Cambridge University Press, 2015.

[2] R. A. Soref and B. R. Bennett, "Electro-optical effects in silicon," IEEE J. Quantum Electron. 23, 123129 (1987). 